\title{
Large-scale structures of scalar and velocity in a turbulent jet flow
}

\author{
Jesse Reijtenbagh*, Jerry Westerweel, Willem van de Water
}

Laboratory for Aero and Hydrodynamics, Delft University of Technology and J.M. Burgers Centre for Fluid Dynamics, 2628 CD Delft, The Netherlands

* j.reijtenbagh@tudelft.nl

We study the relation between large-scale structures in the concentration field with those in the velocity field in a dye-seeded turbulent jet. The scalar concentration in a plane is measured using laser-induced fluorescence. Uniform concentration zones of an advected scalar are identified using cluster analysis. We simultaneously measure the two-dimensional velocity field using particle image velocimetry. The structures in the velocity field are characterized by finite-time Lyapunov exponents. The measurement of the scalarand velocity fields moves with the mean flow. In this moving frame, turbulent structures remain in focus long enough to observe well-defined ridges of the finite-time Lyapunov field. This field gauges the rate of point separation along Lagrangian trajectories; it was measured both for future and past times since the instant of observation. The edges of uniform concentration zones are correlated with the ridges of the past-time Lyapunov field, but not with those of the future-time Lyapunov field.

(a)

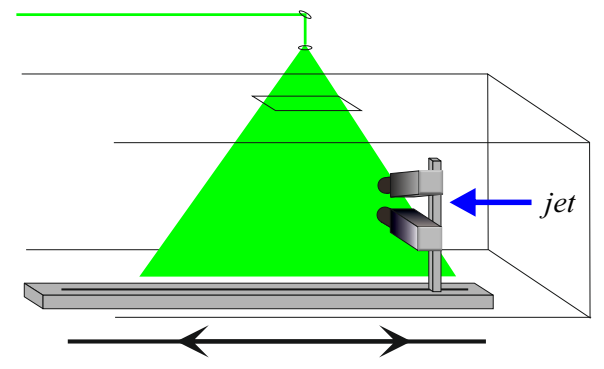

(b)

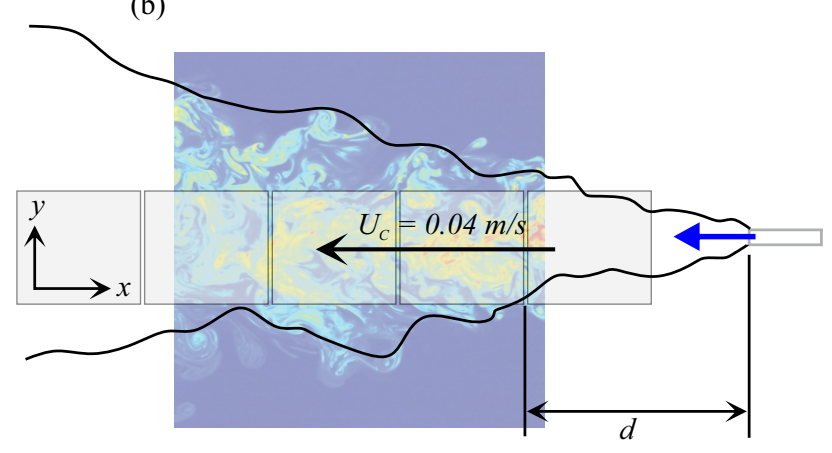

Figure 1: The experimental setup. (a) Schematic view of the arrangement of cameras,traversing system and jet (not to scale). (b) Schematic view of the turbulent jet and sketch of the co-moving observation window (not to scale). In each run a total of 210 PIV frames and $101 \mathrm{LIF}$ frames is acquired at a rate of $10 \mathrm{~Hz}$. The coordinates within a comoving frame are $(x, y)$, the distance of its right edge to the orifice is $d$.

The experiments involve a turbulent jet which contains a fluorescent dye (Rhodamine B) and is seeded with PIV-particles. In a laboratory fixed frame (Eulerian frame), the observation of Lagrangian dynamics is limited by the passage of flow structures through the observation window. Therefore, an experiment was designed where the measuring equipment moves with the mean flow, by using a traverse mechanism driven by a stepper motor, as demonstrated in figure 1. Separate cameras were used for particle image velocimetry and laser induced fluorescence to measure the velocity- and concentration field in a thin slice of the evolving turbulent jet. The PIV-camera is equipped with a shortpass filter (FES0550, Thorlabs, $<550 \mathrm{~nm}$ ) with a transmission ratio in the rejection region of $0.01 \%$ and a $105 \mathrm{~mm}$ lens, while the LIF camera has a longpass filter (FEL0550, Thorlabs, $>550 \mathrm{~nm}$ ) with a transmission ratio in the rejection region of $0.01 \%$, and also a $105 \mathrm{~mm}$ lens. Both the PIV- and LIF-measurements are done using a double-pulsed Nd:YAG laser (Spectra Physics Quanta Ray). The velocities are found from the images of the PIV-camera using DaVis, while the scalar concentration from the LIF-images is found using a third-order calibration function based on the image intensity and the position of this scalar. This calibration function is determined by performing LIF-measurements on a container with known dye concentrations that covers the entire measurement range.

In snapshots of the concentration field we identify regions with more-or-less uniform concentration, using the methods of cluster analysis, recently introduced in turbulence by Fan et al. (2019) (figure 2). 
These uniform concentration zones are reminiscent of uniform momentum zones that are found in turbulent shear flows (de Silva et al., 2016; Eisma et al., 2015; Adrian et al., 2000). We find the edges of these regions, which we link to structures of the velocity field. These structures are defined by the spreading rate of two nearby fluid parcels, quantified by the finite-time Lyapunov exponent (FTLE). Local maxima of this Lyapunov exponent are possible candidates of Lagrangian coherent structures (LCS), which form transfer barriers in the flow (Shadden et al. 2005). Lyapunov exponents can be found by looking at the spreading rate of two fluid parcels in both forward an backward in time, which results in past FTLE and future FTLE, respectively. By moving our measurement equipment along with the flow, we greatly increase the time fluid parcels can be observed, which results in a more detailed FTLE-field.
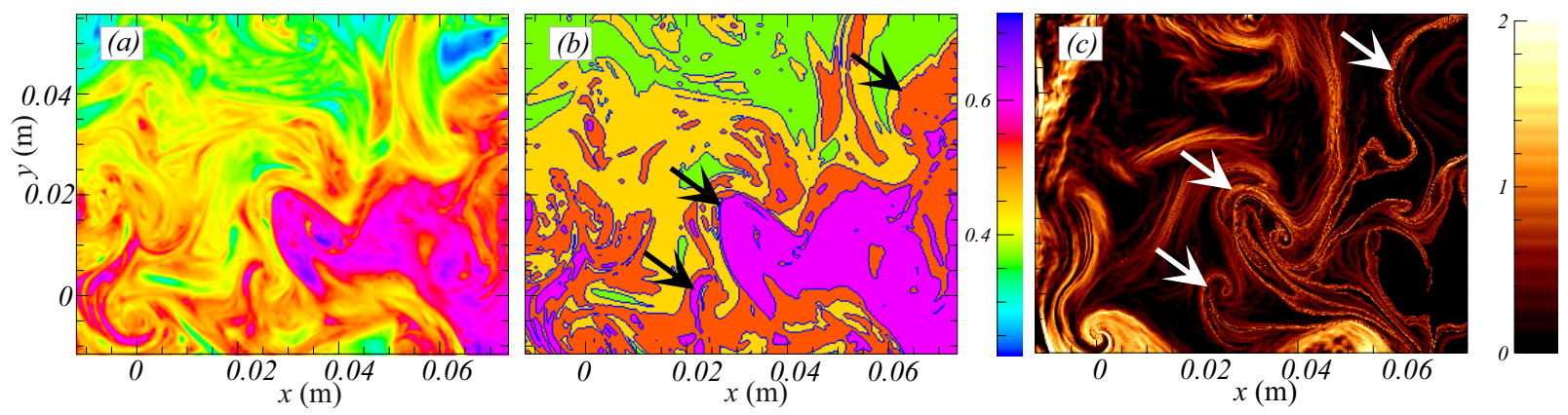

Figure 2: (a) Snapshot of tracer concentration $c(x, t)$, taken at $d=0.83 m$ from the jet orifice. (b) Field of (a), coarsened into 4 uniform concentration zones. (c) Past finite-time Lyapunov exponent in the same field as (a) and (b). The large-scale filamentous structure of $\Lambda_{-T}(x, y)$ roughly corresponds to the large-scale structure of the edges of the uniform concentration zones (suggested by arrows).

Comparing both the past and future FTLE-field with the edges of the uniform concentration zones shows similar structures in the concentration-field and the past FTLE-field (figure 2). These similarities are not found between the future FTLE and the uniform concentration zones. Although the structure of the past FTLE-field resembles that of the edges of concentration zones, they do not coincide exactly. The normalized correlation between edges of concentration zones and the past FTLE-field is small $(\approx 0.02)$, and the conditional averages of the FTLE-fields on lines perpendicular to zone edges only show a small peak of the past FTLE on the edge of a uniform concentration zone.

\section{References}

Adrian RJ, Meinhart CD, and Tomkins CD (2000) Vortex organization in the outer region of the turbulent boundary layer. J Fluid Mech 422:1-54

de Silva CM, Hutchins N, and Marusic I (2016) Uniform momentum zones in turbulent boundary layers. $J$ Fluid Mech 786:309-331

Eisma J, Westerweel J, Ooms G, and Elsinga GE (2015) Interfaces and internal layers in a turbulent boundary layer. Phys Fluids 27

Fan DS, Xu JL, Yao MX, and Hickey JP (2019) On the detection of internal interfacial layers in turbulent flows. Journal of Fluid Mechanics 872:198-217

Shadden SC, Lekien F, and Marsden JE (2005) Definition and properties of Lagrangian coherent structures from finite-time Lyapunov exponents in two-dimensional aperiodic flows. Phys D Nonlinear Phenom 212:271-304 